TRANSACTIONS OF THE

AMERICAN MATHEMATICAL SOCIETY

Volume 351 , Number 12, Pages 4713-4726

S 0002-9947(99)02523-4

Article electronically published on August 25, 1999

\title{
HOMOGENEOUS SPACES WITH INVARIANT PROJECTIVELY FLAT AFFINE CONNECTIONS
}

\author{
HIROHIKO SHIMA
}

\begin{abstract}
We characterize invariant projectively flat affine connections in terms of affine representations of Lie algebras, and show that a homogeneous space admits an invariant projectively flat affine connection if and only if it has an equivariant centro-affine immersion. We give a correspondence between semi-simple symmetric spaces with invariant projectively flat affine connections and central-simple Jordan algebras.
\end{abstract}

\section{INTRODUCTION}

An affine connection is said to be projectively flat if it is locally projectively equivalent to a flat affine connection. In this paper we study invariant projectively flat affine connections.

Applying the theory of projective normal Cartan connections [A] gave a correspondence between the set of invariant projectively flat affine connections on $G / K$ and the set of projective equivalence classes of Lie algebra homomorphisms from the Lie algebra $\mathfrak{g}$ of $G$ to $\mathfrak{s l}(n+1, \mathbf{R})$ where $n=\operatorname{dim} G / K$. Using this correspondence [A] classified irreducible classical Riemannian symmetric spaces with invariant projectively flat affine connections.

The following facts are fundamental for projectively flat affine connections [NS]:

A. A torsion-free and Ricci-symmetric affine connection $D$ on an n-dimensional manifold is projectively flat if and only if

(i) the curvature tensor $R$ and the Ricci tensor Ric satisfy

$$
R(X, Y) Z=\frac{1}{n-1}\{\operatorname{Ric}(Y, Z) X-\operatorname{Ric}(X, Z) Y\},
$$

(ii) the Ricci tensor satisfies the Codazzi equation, that is,

$$
\left(D_{X} \operatorname{Ric}\right)(Y, Z)=\left(D_{Y} \operatorname{Ric}\right)(X, Z) .
$$

B. The induced connections of centro-affine hypersurface immersions are projectively flat.

Being motivated the above facts $[\mathrm{NP}]$ gave a correspondence between Lie groups admitting bi-invariant projectively flat affine connections and associative algebras with unit, and classified all such spaces.

Received by the editors March 15, 1996.

1991 Mathematics Subject Classification. Primary 53C05, 53C30, 53C35, 53A15; Secondary $17 \mathrm{C} 20$.

Key words and phrases. Invariant projectively flat affine connections, centro-affine immersions, homogeneous spaces, symmetric spaces, Jordan algebras.

(C)1999 American Mathematical Society 
Along the same line as in $[\mathrm{NP}]$, in section 1 we relate the existence of invariant projectively flat affine connections to that of affine representations of Lie algebras due to $[\mathrm{Ks}]$. As an immediate consequence we find that a homogeneous space $G / K$ admits an invariant projectively flat affine connection if and only if $G / K$ has an equivariant centro-affine hypersurface immersion. It seems that our method is more elementary and direct than in $[\mathrm{A}]$. In section 2 we give a correspondence between $n$-dimensional Lie groups with left invariant projectively flat affine connections and $(n+1)$-dimensional left symmetric algebras with unit. In section 3 we show that semi-simple symmetric spaces with invariant projectively flat affine connections correspond to central-simple Jordan algebras and are realized as centro-affine hypersurfaces in the algebras (cf. [Ka]). Riemannian semi-simple symmetric spaces with invariant projectively flat affine connections correspond to simple formal real Jordan algebras. The classification of central-simple Jordan algebras and simple formal real Jordan algebras were given in [BK]. In section 4 for a better understanding we explain our correspondence by typical examples.

\section{ACKNOWLEDGMENT}

This work was done while I was staying at Brown University. I would like to express my sincere thanks to Professor K. Nomizu, who kindly acquainted me with the importance of projectively flat affine connections.

\section{INVARIANT PROJECTIVELY FLAT AFFINE CONNECTIONS}

Let $G$ be a simply connected Lie group and $K$ a connected closed subgroup of $G$. Assume that $G$ acts effectively on $G / K$. We denote by $\mathfrak{g}$ and $\mathfrak{k}$ the Lie algebras of $G$ and $K$, respectively. We enlarge $\mathfrak{g}$ as follows

$$
\begin{aligned}
& \tilde{\mathfrak{g}}=\mathfrak{g} \oplus \mathbf{R} E, \\
& {[\tilde{\mathfrak{g}}, E]=\{0\} .}
\end{aligned}
$$

Then our first main result is the following.

Theorem 1.1. An n-dimensional simply connected effective homogeneous space $G / K$ admits a $G$-invariant projectively flat affine connection if and only if $\tilde{\mathfrak{g}}$ has an affine representation $(\tilde{f}, \tilde{q})$ on an $(n+1)$-dimensional real vector space $\tilde{V}$, that is,

(i) $\tilde{f}$ is a representation of $\tilde{\mathfrak{g}}$ on $\tilde{V}$,

(ii) $\tilde{q}$ is a linear mapping from $\tilde{\mathfrak{g}}$ to $\tilde{V}$ such that

$$
\tilde{q}([\tilde{X}, \tilde{Y}])=\tilde{f}(\tilde{X}) \tilde{q}(\tilde{Y})-\tilde{f}(\tilde{Y}) \tilde{q}(\tilde{X}) \quad \text { for } \quad \tilde{X}, \tilde{Y} \in \tilde{\mathfrak{g}}
$$

with the following properties:

(iii) $\tilde{q}$ is surjective and the kernel is $\mathfrak{k}$,

(iv) $\tilde{f}(E)$ is the identity mapping $I_{\tilde{V}}$ of $\tilde{V}$ and $\tilde{q}(E) \neq 0$.

Corollary 1.2. Let $G / K$ be a simply connected homogeneous space. Then the following conditions are equivalent.

(i) $G / K$ admits an invariant projectively flat affine connection.

(ii) $G / K$ has an equivariant centro-affine hypersurface immersion into a real affine space. 
Proof of Theorem 1.1. Suppose that $G / K$ admits a $G$-invariant projectively flat affine connection $D$. For $X \in \mathfrak{g}$, we denote by $X^{*}$ a vector field on $G / K$ induced by $\exp (-t X)$. Set

$$
A_{X^{*}} Y^{*}=-D_{Y^{*}} X^{*} .
$$

Then it is known

$$
A_{X^{*}} Y^{*}=\left(L_{X^{*}}-D_{X^{*}}\right) Y^{*},
$$

where $L_{X^{*}}$ denote Lie differentiation by $X^{*}$, and

$$
A_{[X, Y]^{*}}=\left[A_{X^{*}}, A_{Y^{*}}\right]-R\left(X^{*}, Y^{*}\right),
$$

where $R$ is the curvature tensor for $D$ [KN, p. 235]. Let $V$ be the tangent space of $G / K$ at $o=\{K\}$. We set

$$
\begin{aligned}
& f(X)=\left(A_{X^{*}}\right)_{o}, \\
& q(X)=X_{o}^{*},
\end{aligned}
$$

where the subscript $o$ of tensor fields means the values of the tensor fields at $o$. Then we have

$$
\begin{aligned}
& f([X, Y])=[f(X), f(Y)]-R\left(X^{*}, Y^{*}\right)_{o}, \\
& \operatorname{ker} q=\mathfrak{k} .
\end{aligned}
$$

Since $D$ is projectively flat it follows that

$$
R\left(X^{*}, Y^{*}\right)=\gamma\left(Y^{*}, \quad\right) X^{*}-\gamma\left(X^{*}, \quad\right) Y^{*},
$$

where $\gamma=\frac{1}{n-1}$ Ric. Thus we have

$$
f([X, Y])=[f(X), f(Y)]-\gamma_{o}(q(Y), \quad) q(X)+\gamma_{o}(q(X), \quad) q(Y) .
$$

Since the torsion tensor of $R$ vanishes, it follows that

$$
q([X, Y])=f(X) q(Y)-f(Y) q(X) .
$$

In fact,

$$
\begin{aligned}
q([X, Y]) & =\left[X^{*}, Y^{*}\right]_{o} \\
& =\left(-A_{Y^{*}} X^{*}+A_{X^{*}} Y^{*}\right)_{o} \\
& =-f(Y) q(X)+f(X) q(Y) .
\end{aligned}
$$

Since $\gamma$ is $G$-invariant, we obtain

$$
\begin{aligned}
& \left(D_{X^{*}} \gamma\right)\left(Y^{*}, Z^{*}\right) \\
& =X^{*}\left(\gamma\left(Y^{*}, Z^{*}\right)\right)-\gamma\left(D_{X^{*}} Y^{*}, Z^{*}\right)-\gamma\left(Y^{*}, D_{X^{*}} Z^{*}\right) \\
& =\gamma\left(L_{X^{*}} Y^{*}, Z^{*}\right)+\gamma\left(Y^{*}, L_{X^{*}} Z^{*}\right)-\gamma\left(D_{X^{*}} Y^{*}, Z^{*}\right)-\gamma\left(Y^{*}, D_{X^{*}} Z^{*}\right) \\
& =\gamma\left(A_{X^{*}} Y^{*}, Z^{*}\right)+\gamma\left(Y^{*}, A_{X^{*}} Z^{*}\right) .
\end{aligned}
$$

Using the Codazzi equation for the Ricci tensor,

$$
\left(D_{X^{*}} \gamma\right)\left(Y^{*}, Z^{*}\right)=\left(D_{Y^{*}} \gamma\right)\left(X^{*}, Z^{*}\right)
$$

we have

$$
\begin{aligned}
& \gamma_{o}(f(X) q(Y), q(Z))+\gamma_{o}(q(Y), f(X) q(Z)) \\
& =\gamma_{o}(f(Y) q(X), q(Z))+\gamma_{o}(q(X), f(Y) q(Z)) .
\end{aligned}
$$


We enlarge the vector space $V$ so that

$$
\tilde{V}=V \oplus \mathbf{R} e .
$$

For $X \in \mathfrak{g}$ we define an endomorphism $\tilde{f}(X)$ of $\tilde{V}$ by

$$
\begin{aligned}
& \tilde{f}(X) q(Z)=f(X) q(Z)-\gamma_{o}(q(X), q(Z)) e, \\
& \tilde{f}(X) e=q(X) .
\end{aligned}
$$

Using (1.1), (1.2) and (1.3), we have

$$
\begin{aligned}
{[} & \tilde{f}(X), \tilde{f}(Y)] q(Z) \\
= & {[f(X), f(Y)] q(Z)-\gamma_{o}(q(Y), q(Z)) q(X)+\gamma_{o}(q(X), q(Z)) q(Y) } \\
& -\left\{\gamma_{o}(q(X), f(Y) q(Z))-\gamma_{o}(q(Y), f(X) q(Z))\right\} e \\
= & f([X, Y]) q(Z)-\left\{\gamma_{o}(f(X) q(Y), q(Z))-\gamma_{o}(f(Y) q(X), q(Z))\right\} e \\
= & f([X, Y]) q(Z)-\gamma_{o}(q([X, Y]), q(Z)) e \\
= & \tilde{f}([X, Y]) q(Z),
\end{aligned}
$$

and

$$
\begin{aligned}
& {[\tilde{f}(X), \tilde{f}(Y)] e} \\
& =\tilde{f}(X) q(Y)-\tilde{f}(Y) q(X)=f(X) q(Y)-f(Y) q(X)=q([X, Y]) \\
& =\tilde{f}([X, Y]) e .
\end{aligned}
$$

These imply that $(\tilde{f}, q)$ is an affine representation of $\mathfrak{g}$ on $\tilde{V}$, that is,

$$
\begin{aligned}
\tilde{f}([X, Y]) & =[\tilde{f}(X), \tilde{f}(Y)], \\
q([X, Y]) & =\tilde{f}(X) q(Y)-\tilde{f}(Y) q(X),
\end{aligned}
$$

for $X, Y \in \mathfrak{g}$. We extend this affine representation $(\tilde{f}, q)$ of $\mathfrak{g}$ by

$$
\begin{aligned}
& \tilde{f}(\tilde{X})= \begin{cases}\tilde{f}(X), & \tilde{X}=X \in \mathfrak{g}, \\
I_{\tilde{V}}, & \tilde{X}=E,\end{cases} \\
& \tilde{q}(\tilde{X})= \begin{cases}q(X), & \tilde{X}=X \in \mathfrak{g}, \\
e, & \tilde{X}=E .\end{cases}
\end{aligned}
$$

Then $(\tilde{f}, \tilde{q})$ is an affine representation of $\tilde{\mathfrak{g}}$ on $\tilde{V}$ with required properties.

Conversely, suppose that $\tilde{\mathfrak{g}}$ admits an affine representation $(\tilde{f}, \tilde{q})$ on $\tilde{V}$ satisfying (iii)(iv). Using an affine coordinate system $\left\{x^{1}, \ldots, x^{n+1}\right\}$ on $\tilde{V}$ we can express an affine mapping $\tilde{v} \longrightarrow \tilde{f}(\tilde{X}) \tilde{v}+\tilde{q}(\tilde{X})$ by an $(n+2) \times(n+2)$ matrix representation

$$
a(\tilde{X})=\left[\begin{array}{cc}
\tilde{f}(\tilde{X})_{j}^{i} & \tilde{q}(\tilde{X})^{i} \\
0 & 0
\end{array}\right]
$$

where $\left[\tilde{f}(\tilde{X})_{j}^{i}\right]$ is an $(n+1) \times(n+1)$ matrix and $\left[\tilde{q}(\tilde{X})^{i}\right]$ is a $(n+1)$ row vector. Then $\tilde{X} \longrightarrow a(\tilde{X})$ is an injective Lie algebra homomorphism from $\tilde{\mathfrak{g}}$ into the Lie algebra of all $(n+2) \times(n+2)$ matrices. We set $\tilde{\mathfrak{g}}_{a}=a(\tilde{\mathfrak{g}}), \mathfrak{g}_{a}=a(\mathfrak{g})$ and $\mathfrak{c}_{a}=a(\mathbf{R} E)$. We denote by $\tilde{G}_{a}, G_{a}$ and $C_{a}$ the linear Lie subgroup of $G L(n+2, \mathbf{R})$ generated by $\tilde{\mathfrak{g}}_{a}$, $\mathfrak{g}_{a}$ and $\mathfrak{c}_{a}$, respectively. An element $\tilde{s} \in \tilde{G}_{a}$ is expressed by

$$
\tilde{s}=\left[\begin{array}{cc}
\tilde{\mathbf{f}}(\tilde{s}) & \tilde{\mathbf{q}}(\tilde{s}) \\
0 & 1
\end{array}\right]
$$


where $\tilde{\mathbf{f}}(\tilde{s})$ and $\tilde{\mathbf{q}}(\tilde{s})$ are the linear part and the translation part of $\tilde{s}$, respectively. Let $\tilde{\Omega}_{a}$ and $M_{a}$ be the orbit of $\tilde{G}_{a}$ and $G_{a}$ through the origin $o$ respectively. Then we have

$$
\begin{aligned}
& \tilde{\Omega}_{a}=\tilde{\mathbf{q}}\left(\tilde{G}_{a}\right)=C_{a} G_{a} / K_{a}=C_{a} M_{a}, \\
& M_{a}=\tilde{\mathbf{q}}\left(G_{a}\right)=G_{a} / K_{a},
\end{aligned}
$$

where $K_{a}=\left\{s \in G_{a} \mid \tilde{\mathbf{q}}(s)=0\right\}$, and its Lie algebra is $a(\mathfrak{k})$. Since $\tilde{q}(\tilde{\mathfrak{g}})=\tilde{V}, \tilde{\Omega}_{a}$ is an open orbit in $\tilde{V}$. For $\tilde{X} \in \tilde{\mathfrak{g}}$ we denote by $\tilde{X}^{*}$ a vector field on $\tilde{\Omega}_{a}$ induced by $\exp a(-t \tilde{X})$. Since $\tilde{\Omega}_{a}=C_{a} M_{a}$ is an open set, a curve $\exp a(-t E) m$ through $m \in M_{a}$ is transversal to $M_{a}$ at $m$. Hence $E^{*}$ is transversal to $M_{a}$.

Let $\tilde{D}$ be the canonical flat affine connection on $\tilde{V}$. As in affine differential geometry [NS], we can define the induced affine connection $D$ on $M_{a}$ and the affine fundamental form $h$ by

$$
\tilde{D}_{X^{*}} Y^{*}=D_{X^{*}} Y^{*}+h\left(X^{*}, Y^{*}\right) E^{*},
$$

for $X, Y \in \mathfrak{g}$. Then, $D$ and $h$ are invariant by $G_{a}$, because $\tilde{D}$ and $E^{*}$ are invariant by $\tilde{G}_{a}$. Since $E^{*}=-\sum_{i}\left(x^{i}+\tilde{q}^{i}(E)\right) \partial / \partial x^{i}, M_{a}$ is a centro-affine hypersurface with center $-\tilde{q}(E)$. Hence the induced connection $D$ is projectively flat [NS]. Since $G$ is simply connected, there exists a covering homomorphism

$$
\rho: G \longrightarrow G_{a}
$$

such that $d \rho(X)=a(X)$. K being the identity component of $\rho^{-1}\left(K_{a}\right)$, we have a covering mapping

$$
G / K \longrightarrow G / \rho^{-1}\left(K_{a}\right) \cong G_{a} / K_{a}
$$

induced by $\rho$. Hence $G / K$ admits a $G$-invariant projectively flat affine connection.

Proof of Corollary 1.2. (i) $\Longrightarrow$ (ii) follows from the above arguments. The induced affine connection of a centro-affine immersion being projectively flat [NS], we have (ii) $\Longrightarrow$ (i).

\section{The CASE of Lie groups}

Let $V$ be an algebra over $\mathbf{R}$ with multiplication $u v$. We set

$$
[u v w]=u(v w)-(u v) w .
$$

If the algebra $V$ satisfies

$$
[u v w]=[v u w]
$$

then $V$ is said to be a left symmetric algebra [V2]. The following theorem was essentially known to Koszul and Vinberg.

Theorem 2.1. There is a natural one-one correspondence between

(i) n-dimensional simply connected Lie groups with left invariant flat affine connections up to affine diffeomorphism;

(ii) n-dimensional left symmetric algebras over $\mathbf{R}$ up to algebraic isomorphism.

In this section we prove the following.

Theorem 2.2. There is a natural one-one correspondence between

(i) n-dimensional simply connected Lie groups with left invariant projectively flat affine connections up to equivariant projective diffeomorphism; 
(ii) $(n+1)$-dimensional left symmetric algebras over $\mathbf{R}$ with unit up to algebraic isomorphism.

Proof. Using the same notation as in section 1 we can find by Theorem 1.1 an affine representation $(\tilde{f}, \tilde{q})$ of the Lie algebra $\tilde{\mathfrak{g}}$ on an $(n+1)$-dimensional real vector space $\tilde{V}$ satisfying the conditions (iii)(iv). Since $\tilde{q}: \tilde{\mathfrak{g}} \longrightarrow \tilde{V}$ is an isomorphism we define a multiplication law in $\tilde{V}$ by

$$
u v=\tilde{f}\left(\tilde{q}^{-1}(u)\right) v .
$$

Denoting by $L_{u}$ the left multiplication by $u$ we have

$$
\begin{aligned}
& {\left[L_{u}, L_{v}\right]=L_{u v-v u},} \\
& u e=e u=u,
\end{aligned}
$$

where $e=\tilde{q}(E)$. In fact, since

$$
\tilde{q}\left(\left[\tilde{q}^{-1}(u), \tilde{q}^{-1}(v)\right]\right)=\tilde{f}\left(\tilde{q}^{-1}(u)\right) v-\tilde{f}\left(\tilde{q}^{-1}(v)\right) u=u v-v u,
$$

we have

$$
\begin{aligned}
L_{u v-v u} & =\tilde{f}\left(\tilde{q}^{-1}(u v-v u)\right)=\tilde{f}\left(\left[\tilde{q}^{-1}(u), \tilde{q}^{-1}(v)\right]\right) \\
& =\left[\tilde{f}\left(\tilde{q}^{-1}(u)\right), \tilde{f}\left(\tilde{q}^{-1}(v)\right)\right]=\left[L_{u}, L_{v}\right],
\end{aligned}
$$

and

$$
u e=e u+\tilde{q}\left(\left[\tilde{q}^{-1}(u), \tilde{q}^{-1}(e)\right]\right)=e u=\tilde{f}(E) u=u .
$$

By (2.2) we have

$$
[u v w]=[v u w] .
$$

Thus the algebra $\tilde{V}$ is an $(n+1)$-dimensional left symmetric algebra with unit $e$.

Conversely suppose that $\tilde{V}$ is an $(n+1)$-dimensional left symmetric algebra with unit $e$. Let $V=\left\{v \in \tilde{V} \mid \operatorname{Tr} L_{v}=0\right\}$. Since $\tilde{v}-\left\{1 /(n+1) \operatorname{Tr} L_{\tilde{v}}\right\} e \in V$, it follows that $\tilde{V}=V \oplus \mathbf{R} e$. We set

$$
\begin{aligned}
& \mathfrak{g}(V)=\left\{L_{v} \mid v \in V\right\}, \\
& \mathfrak{g}(\tilde{V})=\left\{L_{\tilde{v}} \mid \tilde{v} \in \tilde{V}\right\} .
\end{aligned}
$$

Then by $(2.2), \mathfrak{g}(V)$ and $\mathfrak{g}(\tilde{V})$ are Lie algebras, and we have

$$
\mathfrak{g}(\tilde{V})=\mathfrak{g}(V) \oplus \mathbf{R} I_{\tilde{V}} .
$$

Setting $\tilde{f}\left(L_{\tilde{v}}\right)=L_{\tilde{v}}$ and $\tilde{q}\left(L_{\tilde{v}}\right)=\tilde{v}$, we obtain an affine representation $(\tilde{f}, \tilde{q})$ of $\mathfrak{g}(\tilde{V})$ satisfying the conditions (iii)(iv) of Theorem 1.1. Thus the simply connected Lie group with Lie algebra $\mathfrak{g}(V)$ admits a left invariant projectively flat affine connection.

Remark. [NP] gave a correspondence between $n$-dimensional Lie groups with biinvariant projectively flat affine connections and $(n+1)$-dimensional associative algebras with unit, and classified all such spaces. 


\section{The CASE OF SYMMETRIC SPACES}

In this section we give a correspondence between semi-simple symmetric spaces with invariant projectively flat affine connections and central-simple Jordan algebras with unit.

Let $(G, K)$ be an effective symmetric pair where $G$ is semi-simple and let $\mathfrak{g}=\mathfrak{k}+\mathfrak{m}$ be the canonical decomposition, that is,

$$
[\mathfrak{k}, \mathfrak{k}] \subset \mathfrak{k}, \quad[\mathfrak{k}, \mathfrak{m}] \subset \mathfrak{m}, \quad[\mathfrak{m}, \mathfrak{m}] \subset \mathfrak{k} .
$$

Suppose that $G / K$ admits a $G$-invariant projectively flat affine connection. As in section 1 we enlarge $\mathfrak{g}$ so that

$$
\begin{aligned}
& \tilde{\mathfrak{g}}=\mathfrak{g} \oplus \mathbf{R} E, \\
& {[\tilde{\mathfrak{g}}, E]=\{0\},}
\end{aligned}
$$

and set $\tilde{\mathfrak{k}}=\mathfrak{k}, \tilde{\mathfrak{m}}=\mathfrak{m} \oplus \mathbf{R} E$. Then

$$
[\tilde{\mathfrak{k}}, \tilde{\mathfrak{m}}] \subset \tilde{\mathfrak{m}}, \quad[\tilde{\mathfrak{m}}, \tilde{\mathfrak{m}}] \subset \tilde{\mathfrak{k}}
$$

By Theorem 1.1 there exists an affine representation $(\tilde{f}, \tilde{q})$ of $\tilde{\mathfrak{g}}$ on an $(n+1)$ dimensional real vector space $\tilde{V}$ where $n=\operatorname{dim} G / K$. The restriction of $\tilde{q}$ to $\tilde{\mathfrak{m}}$ being an isomorphism, for each $u \in \tilde{V}$ there exists a unique element $X_{u} \in \tilde{\mathfrak{m}}$ such that $\tilde{q}\left(X_{u}\right)=u$. We put

$$
L_{u}=\tilde{f}\left(X_{u}\right)
$$

and define a multiplication law in $\tilde{V}$ by

$$
u \cdot v=L_{u} v .
$$

Then the algebra $\tilde{V}$ is commutative and has unit $e=\tilde{q}(E)$. In fact

$$
\begin{aligned}
& u \cdot v-v \cdot u=\tilde{f}\left(X_{u}\right) \tilde{q}\left(X_{v}\right)-\tilde{f}\left(X_{v}\right) \tilde{q}\left(X_{u}\right)=\tilde{q}\left(\left[X_{u}, X_{v}\right]\right)=0, \\
& e \cdot u=\tilde{f}(E) u=u .
\end{aligned}
$$

Lemma 3.1. For $W \in \tilde{\mathfrak{k}}, \tilde{f}(W)$ is a derivation of the algebra $\tilde{V}$.

Proof. Since

$$
\begin{aligned}
& {\left[W, X_{u}\right] \in \tilde{\mathfrak{m}},} \\
& \tilde{q}\left(\left[W, X_{u}\right]\right)=\tilde{f}(W) u-\tilde{f}\left(X_{u}\right) \tilde{q}(W)=\tilde{f}(W) u,
\end{aligned}
$$

we have

$$
\left[W, X_{u}\right]=X_{\tilde{f}(W) u}
$$

Thus we get

$$
\begin{aligned}
(\tilde{f}(W) u) \cdot v & =\tilde{f}\left(\left[W, X_{u}\right]\right) v \\
& =\tilde{f}(W) \tilde{f}\left(X_{u}\right) v-\tilde{f}\left(X_{u}\right) \tilde{f}(W) v \\
& =\tilde{f}(W)(u \cdot v)-u \cdot(\tilde{f}(W) v) .
\end{aligned}
$$

Define a symmetric bilinear form $\tau$ on $\tilde{V}$ by $\tau(u, v)=\operatorname{Tr} L_{u \cdot v}$.

Lemma 3.2. We have

(i) $\left[\left[L_{u}, L_{v}\right], L_{w}\right]=L_{[u \cdot w \cdot v]}$, where $[u \cdot w \cdot v]=u \cdot(w \cdot v)-(u \cdot w) \cdot v$.

(ii) $\tau(u \cdot v, w)=\tau(v, u \cdot w)$. 
Proof. Since

$$
\begin{aligned}
\tilde{q}\left(\left[\left[X_{u}, X_{v}\right], X_{w}\right]\right) & =\tilde{f}\left(\left[X_{u}, X_{v}\right]\right) \tilde{q}\left(X_{w}\right)-\tilde{f}\left(X_{w}\right) \tilde{q}\left(\left[X_{u}, X_{v}\right]\right) \\
& =\left[L_{u}, L_{v}\right] w \\
& =[u \cdot w \cdot v]
\end{aligned}
$$

we have

$$
\left[\left[X_{u}, X_{v}\right], X_{w}\right]=X_{[u \cdot w \cdot v]} .
$$

This implies (i). Using (i) we obtain

$$
\begin{aligned}
\tau(u \cdot v, w)-\tau(v, u \cdot w) & =\operatorname{Tr} L_{(u \cdot v) \cdot w}-\operatorname{Tr} L_{v \cdot(u \cdot w)} \\
& =-\operatorname{Tr} L_{[v \cdot u \cdot w]} \\
& =-\operatorname{Tr}\left[\left[L_{v}, L_{w}\right], L_{u}\right] \\
& =0 .
\end{aligned}
$$

Lemma 3.3. $\tau$ is non-degenerate.

Proof. We set

$$
\tilde{V}_{0}=\left\{v_{0} \in \tilde{V} \mid \tau\left(v_{0}, v\right)=0 \text { for all } v \in \tilde{V}\right\} .
$$

For $v_{0} \in \tilde{V}_{0}, v \in \tilde{V}$ and $W \in \tilde{\mathfrak{k}}$ we have

$$
\tilde{q}\left(\left[W, X_{v_{o} \cdot v}\right]\right)=\tilde{f}(W) \tilde{q}\left(X_{v_{0} \cdot v}\right)-\tilde{f}\left(X_{v_{0} \cdot v}\right) \tilde{q}(W)=\tilde{f}(W)\left(v_{0} \cdot v\right) .
$$

Hence we know

$$
\begin{aligned}
{\left[\tilde{f}(W), \tilde{f}\left(X_{v_{0} \cdot v}\right)\right] } & =\tilde{f}\left(X_{\tilde{f}(W)\left(v_{0} \cdot v\right)}\right) \\
& =L_{\tilde{f}(W)\left(v_{0} \cdot v\right)} \\
& =L_{\left(\tilde{f}(W) v_{0}\right) \cdot v}+L_{v_{0} \cdot(\tilde{f}(W) v)} .
\end{aligned}
$$

Thus we obtain

$$
\begin{aligned}
0 & =\operatorname{Tr}\left[\tilde{f}(W), \tilde{f}\left(X_{v_{0} \cdot v}\right)\right] \\
& =\operatorname{Tr} L_{\left(\tilde{f}(W) v_{0}\right) \cdot v}+\operatorname{Tr} L_{v_{0} \cdot(\tilde{f}(W) v)} \\
& =\tau\left(\tilde{f}(W) v_{0}, v\right) .
\end{aligned}
$$

Hence we get

$$
\tilde{f}(\tilde{\mathfrak{k}}) \tilde{V}_{0} \subset \tilde{V}_{0} .
$$

For $v_{0} \in \tilde{V}_{0}, v \in \tilde{V}$ and $X \in \tilde{\mathfrak{m}}$ we have

$$
\tau\left(\tilde{f}(X) v_{0}, v\right)=\tau\left(\tilde{q}(X) \cdot v_{0}, v\right)=\tau\left(v_{0}, \tilde{q}(X) \cdot v\right)=0 .
$$

This means

$$
\tilde{f}(\tilde{\mathfrak{m}}) \tilde{V}_{0} \subset \tilde{V}_{0}
$$

These show $\tilde{f}(\tilde{\mathfrak{g}}) \tilde{V}_{0} \subset \tilde{V}_{0}$, and

$$
\tilde{f}(\mathfrak{g}) \tilde{V}_{0} \subset \tilde{V}_{0} .
$$

Since $\mathfrak{g}$ is semi-simple, the representation $\tilde{f}$ of $\mathfrak{g}$ on $\tilde{V}$ is completely reducible. 
Therefore there exists a complementary subspace $\tilde{V}_{1}$ of $\tilde{V}$ such that

$$
\begin{gathered}
\tilde{V}=\tilde{V}_{0} \oplus \tilde{V}_{1}, \\
\tilde{f}(\mathfrak{g}) \tilde{V}_{1} \subset \tilde{V}_{1} .
\end{gathered}
$$

Since $\tilde{f}(E)=I_{\tilde{V}}$ we have

$$
\tilde{f}(\tilde{\mathfrak{g}}) \tilde{V}_{i} \subset \tilde{V}_{i} \quad(i=1,2) .
$$

Thus we get

$$
\tilde{V} \cdot \tilde{V}_{i} \subset \tilde{V}_{i} \quad(i=1,2) .
$$

Denoting $e=e_{0}+e_{1}$ where $e_{i} \in \tilde{V}_{i}$ we know

$$
L_{e_{i}} v_{j}=\delta_{i j} v_{j} \text { for } v_{j} \in \tilde{V}_{j},
$$

where $\delta_{i j}$ is Kronecker's delta. Hence $\operatorname{dim} V_{0}=$ trace of $L_{e_{0}}$ on $\tilde{V}_{0}=\operatorname{trace}$ of $L_{e_{0}}$ on $\tilde{V}=\operatorname{Tr} L_{e_{0} \cdot e_{0}}=\tau\left(e_{0}, e_{0}\right)=0$. This implies that $\tau$ is non-degenerate.

Let us recall the definition of Jordan algebra. An algebra $\tilde{V}$ over $\mathbf{R}$ is said to be a Jordan algebra if, for all $u, v \in \tilde{V}$,

$$
\begin{aligned}
u \cdot v & =v \cdot u, \\
u \cdot\left(u^{2} \cdot v\right) & =u^{2} \cdot(u \cdot v) .
\end{aligned}
$$

The following lemma is due to [V1].

Lemma 3.4. Let $\tilde{V}$ be a commutative algebra with a multiplication $u \cdot v=L_{u} v$. Suppose

(a) $\left[\left[L_{u}, L_{v}\right], L_{w}\right]=L_{[u \cdot w \cdot v]}$,

(b) the bilinear form $\tau(u, v)=\operatorname{Tr} L_{u \cdot v}$ is non-degenerate.

Then $\tilde{V}$ is a semi-simple Jordan algebra.

Therefore our algebra $\tilde{V}$ is a semi-simple Jordan algebra.

Lemma 3.5. The representation $\tilde{f}$ of $\tilde{\mathfrak{g}}$ on $\tilde{V}$ is faithful.

Proof. We set

$$
\operatorname{ker}_{\mathfrak{g}} \tilde{f}=\{X \in \mathfrak{g} \mid \tilde{f}(X)=0\} .
$$

We denote by $d_{\tilde{f}}$ the coboundary operator for the cohomology of the Lie algebra $\mathfrak{g}$ with coefficients in $(\tilde{V}, \tilde{f})$. Regarding $\tilde{q}$ as a 1-dimensional $(\tilde{V}, \tilde{f})$-cochain, we have $\left(d_{\tilde{f}} \tilde{q}\right)(X, Y)=\tilde{f}(X) \tilde{q}(Y)-\tilde{f}(Y) \tilde{q}(X)-\tilde{q}([X, Y])=0$ for $X, Y \in \mathfrak{g}$. Since $\mathfrak{g}$ is semi-simple, there exists an element $\tilde{e} \in \tilde{V}$ such that $\tilde{q}=d_{\tilde{f}} \tilde{e}$. Thus we have

$$
\tilde{q}(X)=\tilde{f}(X) \tilde{e} \text { for } X \in \mathfrak{g} .
$$

This shows that $\operatorname{ker}_{\mathfrak{g}} \tilde{f} \subset \tilde{\mathfrak{k}}=\mathfrak{k}$. By effectiveness we have $\operatorname{ker}_{\mathfrak{g}} \tilde{f}=\{0\}$. Suppose $\tilde{f}(\tilde{X})=0$, where $\tilde{X}=X+x E(X \in \mathfrak{g})$. Then $\operatorname{Tr} \tilde{f}(X)=\operatorname{Tr}(-x \tilde{f}(E))=-x \operatorname{dim} \tilde{V}$. $\mathfrak{g}$ being semi-simple we have $X \in[\mathfrak{g}, \mathfrak{g}]$, and so $\operatorname{Tr} \tilde{f}(X)=0$. Thus $x=0$ and $X \in \operatorname{ker}_{\mathfrak{g}} \tilde{f}=\{0\}$. Hence $\tilde{X}=0$. 
Let $\mathfrak{m}(\tilde{V})=\left\{L_{v} \mid v \in \tilde{V}\right\}$ and let $\mathfrak{k}(\tilde{V})$ be the vector subspace spanned by $\left[L_{u}, L_{v}\right] \quad(u, v \in \tilde{V})$. Then $\tilde{f}(\tilde{\mathfrak{m}})=\mathfrak{m}(\tilde{V})$, and

$$
\mathfrak{g}(\tilde{V})=\mathfrak{k}(\tilde{V})+\mathfrak{m}(\tilde{V}) .
$$

is a Lie algebra. By Lemma 3.1 an element in $\tilde{f}(\tilde{\mathfrak{k}})$ is a derivation of $\tilde{V}$. Since a derivation of a semi-simple Jordan algebra is inner [BK], we have

$$
\tilde{f}(\tilde{\mathfrak{k}})=\mathfrak{k}(\tilde{V}) .
$$

Thus

$$
\tilde{f}: \tilde{\mathfrak{g}}=\tilde{\mathfrak{k}}+\tilde{\mathfrak{m}} \longrightarrow \mathfrak{g}(\tilde{V})=\mathfrak{k}(\tilde{V})+\mathfrak{m}(\tilde{V})
$$

is an isomorphism including decompositions.

The center $Z(\tilde{V})$ of a Jordan algebra $\tilde{V}$ is by definition $[\mathrm{BK}]$

$$
Z(\tilde{V})=\{u \in \tilde{V} \mid[u \cdot v \cdot w]=[v \cdot u \cdot w]=[v \cdot w \cdot u]=0 \text { for all } v, w \in \tilde{V}\} .
$$

A Jordan algebra $\tilde{V}$ with unit $e$ is said to be central-simple if $\tilde{V}$ is simple and $Z(\tilde{V})=\mathbf{R} e$.

Lemma 3.6. Our Jordan algebra $\tilde{V}$ is central-simple.

Proof. Let $c \in Z(\tilde{V})$. Then $0=[c \cdot v \cdot u]=\left[L_{c}, L_{u}\right] v$ for all $u, v \in \tilde{V}$. Thus $\left[L_{c}, L_{u}\right]=0$ for all $u \in \tilde{V}$. This together with (3.1) shows that $L_{c}$ is contained in the center of $\mathfrak{g}(\tilde{V})$. By (3.2) the center of $\mathfrak{g}(\tilde{V})$ is equal to $\tilde{f}$ (the center of $\tilde{\mathfrak{g}}$ ). Since the center of $\tilde{\mathfrak{g}}$ is $\mathbf{R} E$, we know $L_{c} \in \tilde{f}(\mathbf{R} E)=\mathbf{R} L_{e}$. Thus $Z(\tilde{V})=\mathbf{R} e$. $\tilde{V}$ being semi-simple we have a direct sum decomposition

$$
\tilde{V}=\tilde{V}_{1} \oplus \cdots \oplus \tilde{V}_{k}
$$

where $\tilde{V}_{i}$ are simple ideals of $\tilde{V}$. Let us denote $e=e_{1}+\cdots+e_{k}$ where $e_{i} \in \tilde{V}_{i}$. Suppose $\tilde{V}_{1} \neq\{0\}$. Then $e_{1}$ is the unit of $\tilde{V}_{1}$. Let $c_{1} \neq 0 \in Z\left(\tilde{V}_{1}\right)$. We have

$$
\begin{aligned}
& {\left[c_{1} \cdot \tilde{V}_{1} \cdot \tilde{V}_{1}\right]=\{0\},} \\
& {\left[c_{1} \cdot \tilde{V}_{i} \cdot \tilde{V}_{j}\right]=\{0\}, \text { if } i \neq 1 \text { or } j \neq 1 .}
\end{aligned}
$$

Thus

$$
\left[c_{1} \cdot \tilde{V} \cdot \tilde{V}\right]=\{0\}
$$

Analogously we have

$$
\left[\tilde{V} \cdot c_{1} \cdot \tilde{V}\right]=\{0\}, \quad\left[\tilde{V} \cdot \tilde{V} \cdot c_{1}\right]=\{0\} .
$$

Thus $c_{1} \in Z(\tilde{V})=\mathbf{R} e$, and $c_{1}=a e$ where $a \neq 0$. This means that $\tilde{V}_{i}=\{0\}$ if $i \neq 1$. Hence $\tilde{V}$ is simple.

Summing up the above results we have

Theorem 3.7. Let $(G, K)$ be an effective symmetric pair where $G$ is semi-simple. Suppose that the space $G / K$ admits a $G$-invariant projectively flat affine connection. Then there exists a central-simple Jordan algebra $\tilde{V}$ with unit e such that

(i) $\tilde{V}=V \oplus \mathbf{R} e$ (direct sum as vector spaces).

(ii) Let $\mathfrak{m}(V)=\left\{L_{u} \mid u \in V\right\}$ and let $\mathfrak{k}(V)$ be the vector space spanned by $\left[L_{u}, L_{v}\right]$ where $u, v \in V$. Then $\mathfrak{g}(V)=\mathfrak{k}(V)+\mathfrak{m}(V)$ is a Lie algebra and is isomorphic to the Lie algebra $\mathfrak{g}=\mathfrak{k}+\mathfrak{m}$ of $G$ including decompositions. 
Conversely, we have

Theorem 3.8. Let $\tilde{V}$ be a central-simple Jordan algebra with unit e. We set $V=$ $\left\{v \in \tilde{V} \mid \operatorname{Tr} L_{v}=0\right\}$. Let $\mathfrak{m}(V)=\left\{L_{v} \mid v \in V\right\}$ and let $\mathfrak{k}(V)$ be the vector space spanned by $\left[L_{u}, L_{v}\right]$ for $u, v \in V$. Then $\mathfrak{k}(V)$ and $\mathfrak{g}(V)=\mathfrak{k}(V)+\mathfrak{m}(V)$ are linear Lie algebras. Let $G(V)$ and $K(V)$ be linear Lie groups generated by $\mathfrak{g}(V)$ and $\mathfrak{k}(V)$, respectively. Then $(G(V), K(V))$ is a symmetric pair, where $G(V)$ is a semi-simple Lie group, and $G(V) / K(V)$ admits a $G(V)$-invariant projectively flat affine connection.

Proof. It is known $[\mathrm{BK}]$ that $\mathfrak{g}(V)$ is a semi-simple Lie algebra and

$$
[\mathfrak{k}(V), \mathfrak{m}(V)] \subset \mathfrak{m}(V), \quad[\mathfrak{m}(V), \mathfrak{m}(V)] \subset \mathfrak{k}(V) .
$$

Let $\mathfrak{m}(\tilde{V})=\left\{L_{\tilde{v}} \mid \tilde{v} \in \tilde{V}\right\}$ and let $\mathfrak{k}(\tilde{V})$ be the vector space spanned by $\left[L_{\tilde{u}}, L_{\tilde{v}}\right]$ for $\tilde{u}, \tilde{v} \in \tilde{V}$. We put

$$
\mathfrak{g}(\tilde{V})=\mathfrak{k}(\tilde{V})+\mathfrak{m}(\tilde{V})
$$

Then $\mathfrak{g}(\tilde{V})$ is a Lie algebra and

$$
\mathfrak{g}(\tilde{V})=\mathfrak{g}(V)+\mathbf{R} I_{\tilde{V}}
$$

cf. [BK]. We define a representation $\tilde{f}$ of $\mathfrak{g}(\tilde{V})$ on $\tilde{V}$ by $\tilde{f}(\tilde{X})=\tilde{X}$ for $\tilde{X} \in \mathfrak{g}(\tilde{V})$ and a linear mapping $\tilde{q}$ from $\mathfrak{g}(\tilde{V})$ to $\tilde{V}$ by $\tilde{q}\left(W+L_{\tilde{v}}\right)=\tilde{v}$ for $W \in \mathfrak{k}(\tilde{V}), L_{\tilde{v}} \in \mathfrak{m}(\tilde{V})$. Then $(\tilde{f}, \tilde{q})$ is an affine representation of $\mathfrak{g}(\tilde{V})$ on $\tilde{V}$ satisfying the conditions of Theorem 1.1. Therefore the space $G(V) / K(V)$ admits a $G(V)$-invariant projectively flat affine connection.

Remark. Let $G(\tilde{V})$ denote the linear Lie group generated by $\mathfrak{g}(\tilde{V})$. Then $G(\tilde{V})$ is the identity component of the structure group of $\tilde{V}$, and the orbit $\tilde{\Omega}=G(\tilde{V}) e$ is a $\omega$-domain $[\mathrm{BK}],[\mathrm{K}]$. We have

$$
\tilde{\Omega}=\mathbf{R}^{+} G(V) e=\mathbf{R}^{+} G(V) / K(V) .
$$

Thus $\tilde{\Omega}$ is a cone obtained from $G(V) / K(V)$ by positive dilations at the origin 0 .

Remark. For the classification of central-simple Jordan algebras see [BK].

\section{EXAMPLES}

Using typical examples we explain our correspondence between semi-simple symmetric spaces with invariant projectively flat affine connections and central-simple Jordan algebras.

Example 4.1. Quadratic surface $S O(p, n+1-p) / S O(p, n-p)(0 \leq p \leq n)$.

Denoting by $I_{p}$ the unit matrix of degree $p$ we set

$$
\begin{aligned}
J & =\left[\begin{array}{cc}
-I_{p} & 0 \\
0 & I_{n-p}
\end{array}\right], \\
\tilde{J} & =\left[\begin{array}{cc}
-I_{p} & 0 \\
0 & I_{n+1-p}
\end{array}\right]=\left[\begin{array}{ll}
J & 0 \\
0 & 1
\end{array}\right] .
\end{aligned}
$$

Let $M_{p}^{n}$ be the connected component of the set defined by

$$
\left\{x \in \mathbf{R}^{n+1} \mid{ }^{t} x \tilde{J} x=1\right\}
$$


containing ${ }^{t} e=[0, \cdots, 0,1]$. Then $M_{0}^{n}$ is a sphere, and $M_{n}^{n}$ is a hyperbolic space.

Let

$$
S O(p, n-p)=\left\{s \in S L(n, \mathbf{R}) \mid{ }^{t} s J s=J\right\}
$$

Then we know

$$
M_{p}^{n}=S O(p, n+1-p) / S O(p, n-p) .
$$

The Lie algebra $\mathfrak{o}(p, n-p)$ of $S O(p, n-p)$ is

$$
\mathfrak{o}(p, n-p)=\left\{A \in \mathfrak{g l}(n, \mathbf{R}) \mid{ }^{t} A J+J A=0\right\},
$$

and the Lie algebra $\mathfrak{g}$ of $S O(p, n+1-p)$ is

$$
\mathfrak{o}(p, n+1-p)=\left\{\left[\begin{array}{cc}
A & a \\
-{ }^{t}(J a) & 0
\end{array}\right] \mid A \in \mathfrak{o}(p, n-p), a \in \mathbf{R}^{n}\right\} .
$$

Let $\iota$ be an involutive automorphism of $\mathfrak{g}$ defined by

$$
\iota\left(\left[\begin{array}{cc}
A & a \\
-{ }^{t}(J a) & 0
\end{array}\right]\right)=\left[\begin{array}{cc}
A & -a \\
{ }^{t}(J a) & 0
\end{array}\right] .
$$

Then the canonical decomposition of $\mathfrak{g}$ with respect to $\iota$ is

$$
\begin{aligned}
\mathfrak{g} & =\mathfrak{k}+\mathfrak{m}, \\
\mathfrak{k} & =\left\{\left[\begin{array}{cc}
A & 0 \\
0 & 0
\end{array}\right] \mid A \in \mathfrak{o}(p, n-p)\right\}, \\
\mathfrak{m} & =\left\{\left[\begin{array}{cc}
0 & a \\
-{ }^{t}(J a) & 0
\end{array}\right] \mid a \in \mathbf{R}^{n}\right\} .
\end{aligned}
$$

We set

$$
\begin{aligned}
\tilde{\mathfrak{g}} & =\mathfrak{g} \oplus \mathbf{R} E, \\
\tilde{\mathfrak{m}} & =\mathfrak{m} \oplus \mathbf{R} E,
\end{aligned}
$$

where $E=I_{n+1}$. We define an affine representation $(\tilde{f}, \tilde{q})$ of $\tilde{\mathfrak{g}}$ on $\mathbf{R}^{n+1}$ by $\tilde{f}(\tilde{X})=$ $\tilde{X}, \tilde{q}(\tilde{X})=\tilde{X} e$. Then the affine representation $(\tilde{f}, \tilde{q})$ satisfies the conditions of Theorem 1.1. Using the notation in section 3 , for $\tilde{u}={ }^{t}\left[u_{1}, \cdots, u_{n}, u_{n+1}\right] \in \mathbf{R}^{n+1}$ we have

$$
X_{\tilde{u}}=\left[\begin{array}{cc}
u_{n+1} I_{n} & u \\
-{ }^{t}(J u) & u_{n+1}
\end{array}\right],
$$

where $u={ }^{t}\left[u_{1}, \ldots, u_{n}\right] \in \mathbf{R}^{n}$. Hence

$$
\left[\begin{array}{c}
u \\
u_{n+1}
\end{array}\right] \cdot\left[\begin{array}{c}
v \\
v_{n+1}
\end{array}\right]=X_{\tilde{u}} \tilde{v}=\left[\begin{array}{c}
u_{n+1} v+v_{n+1} u \\
-{ }^{t} u J v+u_{n+1} v_{n+1}
\end{array}\right] \text {. }
$$

Thus the Jordan algebra $\mathbf{R}^{n+1}$ with this multiplication coincides with the Jordan algebra $[X ; \mu, e]$ associated to the bilinear form $\mu(\tilde{u}, \tilde{v})=-{ }^{t} u J v+u_{n+1} v_{n+1}$ on $X=\mathbf{R}^{n+1}$, and $e$ [BK, p. 193]. The corresponding $\omega$-domain $\Omega_{p}^{n+1}$ is a cone in $\mathbf{R}^{n+1}$ given by

$$
\left\{x \in \mathbf{R}^{n+1} \mid{ }^{t} x \tilde{J} x>0\right\},
$$

and $\Omega_{p}^{n+1}=\mathbf{R}^{+} M_{p}^{n}$.

Example 4.2. $S L(n, \mathbf{R}) / S O(p, n-p)(0 \leq p \leq n)$. 
Let $\iota$ be an involutive automorphism of $\mathfrak{g}=\mathfrak{s l}(n, \mathbf{R})$ defined by

$$
\iota(X)=-J^{t} X J
$$

where $J$ is the same as in Example 4.1. Then the canonical decomposition of $\mathfrak{g}$ with respect to $\iota$ is

$$
\begin{aligned}
\mathfrak{g} & =\mathfrak{k}+\mathfrak{m}, \\
\mathfrak{k} & =\{A \in \mathfrak{g} \mid \iota(A)=A\}=\mathfrak{o}(p, n-p), \\
\mathfrak{m} & =\{A \in \mathfrak{g} \mid \iota(A)=-A\} .
\end{aligned}
$$

Denoting $E=I_{n}$ we set

$$
\tilde{\mathfrak{g}}=\mathfrak{g} \oplus \mathbf{R} E=\mathfrak{g l}(n, \mathbf{R}) .
$$

Let $\tilde{V}=\left\{v \in \tilde{\mathfrak{g}} \mid{ }^{t} v=v\right\}$. We define an affine representation $(\tilde{f}, \tilde{q})$ of $\tilde{\mathfrak{g}}$ on $\tilde{V}$ by $\tilde{f}(X) v=-{ }^{t} X v-v X, \tilde{q}(X)=-{ }^{t} X J-J X$. Then the affine representation $(\tilde{f}, \tilde{q})$ satisfies the conditions of Theorem 1.1. Using the notation of section 3 we have

$$
X_{u}=-\frac{1}{2} J u, \quad \text { for } u \in \tilde{V}
$$

Hence

$$
u \cdot v=\tilde{f}\left(X_{u}\right) v=\frac{1}{2}(u J v+v J u)
$$

The Jordan algebra with this multiplication is a mutation of the Jordan algebra $\tilde{V}$ with standard multiplication

$$
u \circ v=\frac{1}{2}(u v+v u)
$$

[BK], and the corresponding $\omega$-domain is a cone $\Omega(p, n-p)$ in $\tilde{V}$ consisting of all symmetric matrices of signature $(p, n-p)$. We know

$$
\begin{aligned}
\Omega(p, n-p) & =\left\{{ }^{t} g J g \mid g \in G L(n, \mathbf{R})\right\} \\
& =G L^{+}(n, \mathbf{R}) / S O(p, n-p)=\mathbf{R}^{+} S L(n, \mathbf{R}) / S O(p, n-p),
\end{aligned}
$$

and $S L(n, \mathbf{R}) / S O(p, n-p)$ is a level surface in $\Omega(p, n-p)$ defined by $\operatorname{det} u=1$ (cf. $[\mathrm{Sa}])$.

\section{REFERENCES}

[A] Y. Agaoka, Invariant flat projective structures on homogeneous spaces, Hokkaido Math. J. 11 (1982), 125-172. MR 85g:53038

[BK] H. Braun und M. Koecher, Jordan-Algebren, Grundlehren der Math. Wissenschaften, 128, Springer, Berlin, Heidelberg, New York, 1966. MR 34:4310

[Ka] S. Kaneyuki, The Sylvester's Law of Inertia in Simple Graded Lie Algebras, J. Math. Soc. Japan, 50(1998), 593-614. MR 99f: 17035

[KN] S. Kobayashi and K. Nomizu, Foundations of differential geometry, vol.I, John Wiley \& Sons, New York, 1963. MR 97c:53001b

[K] M. Koecher, Jordan algebras and their applications, Lecture notes, Univ. of Minnesota, Minneapolis, 1962.

[Ks] J.L. Koszul, Domaines bornés homogenes et orbites de groupes de transformations affines, Bull. Soc. Math. France, 89 (1961), 515-533. MR 26:3090

[NP] K. Nomizu and U. Pinkall, On a certain class of homogeneous projectively flat manifolds, Tohoku Math. J. 39 (1987), 407-427. MR 88j:53050

[NS] K. Nomizu and T. Sasaki, Affine Differential Geometry, Cambridge Univ. Press, 1994. MR 96e:53014

[Sa] T. Sasaki, Hyperbolic affine hyperspheres, Nagoya Math. J. 77 (1980), 107-123. MR 81e:53037 
[Sh1] H. Shima, On locally symmetric homogeneous domains of completely reducible linear Lie groups, Math. Ann., 217 (1975), 93-95. MR 52:818

[Sh2] H. Shima, Symmetric spaces with invariant locally Hessian structures, J. Math. Soc. Japan, 29 (1977), 581-589. MR 56:9462

[Sh3] H. Shima, Homogeneous Hessian manifolds, Ann. Inst. Fourier, Grenoble, 30 (1980), 91-128. MR 82a: 53054

[V1] E.B. Vinberg, Homogeneous cones, Dokl. Akad. Nauk SSSR, 133 (1960), 9-12; English transl., Soviet Math. Dokl., 1 (1960), 787-790. MR 25:5077

[V2] E.B. Vinberg, The theory of convex homogeneous cones, Trans. Moscow Math. Soc. 12 (1963), 340-403. MR 28:1637

Department of Mathematics, Yamaguchi University, Yamaguchi 753-8512, Japan

E-mail address: shima@po.cc.yamaguchi-u.ac.jp 